\title{
Assessment of Curve Correction in Cases of Adolescent Idiopathic Scoliosis Operated Through Posterior Approach by Ordinary Pedicle Screws
}

Ibrahim Ahmed Mustafa, Mohammad Abdelmenem Negm, Mohammad Nagah Elnoemany Ashour

Department of Orthopedic Surgery Faculty of Medicine, Al-Azhar University

Corresponding author: Mohammad Nagah Elnoemany Ashour, Mobile: 01017344226, email: m.nagah10391@gmail.com

\begin{abstract}
Background: Curve correction is the main aim in treatment of adolescent idiopathic scoliosis (AIS). Many methods were published for correction and the most common method now is curve correction through posterior approach.

Objective: This study was conducted to assess curve correction in cases of adolescent idiopathic scoliosis that were operated through posterior approach by ordinary pedicle screws.

Patients and methods: This was a retrospective study carried out at Al-Azhar University Hospitals between November 2018 and August 2019. Twenty (20) patients with AIS were treated through posterior approach by ordinary pedicle screw. The patients were cooperative and reliable. The sample was comprised of 17 female and 3 male patients with a mean age of $15.3 \pm 2.9$ years (range 10 - 20 years).

Results: The results were analyzed and compared with the results published by other authors which revealed that SRS 24 questionnaire with an average of 100.2 (of total score 120 representing good outcome). All patients gained height ranged from $3 \mathrm{~cm}$ to $8 \mathrm{~cm}$ with an average of $4.6 \mathrm{~cm}$. Correction of rib hump ranged from $2.2 \mathrm{~cm}$ to $7.4 \mathrm{~cm}$ with an average of $4.5 \mathrm{~cm}$ (about $77 \%$ ).

Conclusion: Finally, we concluded that all pedicle screw constructs are reliable in the treatment of AIS, while longer follow up period is needed.
\end{abstract}

Keywords: Adolescent idiopathic scoliosis, scoliosis research society.

\section{INTRODUCTION}

Scoliosis is a three dimensional deformation of the spine characterized as a sidelong shape of the spine in the coronal plane of more than $10^{\circ}{ }^{(1)}$. It tends to be ordered into three noteworthy sorts: innate, syndromic, and idiopathic. Inherent scoliosis alludes to spinal distortion brought about by unusually framed vertebrae. Syndromic scoliosis is related with a turmoil of the neuromuscular, skeletal, or connective tissue frameworks, neurofibromatosis or other significant ailment. Idiopathic scoliosis has no known reason and can be subdivided dependent on the period of beginning: childish idiopathic scoliosis incorporates patients matured 0-3 years. Adolescent idiopathic scoliosis incorporates patients matured 4-10 years, and youthful idiopathic scoliosis influences individuals matured $>10$ years. Youthful idiopathic scoliosis (AIS) is an auxiliary, parallel, pivoted ebb and flow of the spine that emerges in generally solid kids at or around adolescence. Adolescent idiopathic scoliosis (AIS) is the most widely recognized spinal distortion seen by essential consideration doctors, pediatricians and spinal surgeons ${ }^{(2)}$.

The analysis of AIS is one of prohibition and is made just when different reasons for scoliosis, for example, vertebral distortions, neuromuscular clutters and different disorders have been discounted. As per epidemiological investigations, $1-3 \%$ of kids matured 10-16 years will have some level of spinal ebb and flow, albeit most bends won't require careful intercession ${ }^{(3)}$.

The Scoliosis inquire about society (SRS) prescribes perception in patients who have not arrived at skeletal development and have bends under 25 degrees and in the individuals who are skeletally full grown with bends of under 45 degrees. Sequential radiographs and clinical assessments ought to be gotten at regular intervals until skeletal development and after that at regular intervals a short time later to screen for bend movement into adulthood ${ }^{(4)}$.

Medical procedure is demonstrated for AIS when the Cobb edge is more prominent than 45 degrees or the bend is quickly advancing. The pillar of treatment is spinal combination by means of back (PSF), foremost (ASF), or infrequently consolidated front/back methodology (APSF). The objective is to settle the bend while likewise amending the disfigurement. The most well-known strategy is PSF with pedicle screw and bar obsession, which can be utilized for a wide range of AIS, particularly thoracic and twofold real bends. Back combination was first depicted by Hibbs in 1911 as a method for restricting spinal deformation in the setting of tuberculosis ${ }^{(5)}$.

The target of this investigation was to survey the bend rectification in instances of AIS that was corrected through back methodology utilizing normal pedicle screws.

\section{AIM OF THE WORK}

The aim of this work was to assess curve correction in cases of adolescent idiopathic scoliosis that was operated through posterior approach by ordinary pedicle screws.

\section{PATIENTS AND METHODS}

This was a review study done at Al-Azhar University Hospitals between November 2018 and August 2019.

Ethical approval:

After obtaining approval of ethics committee, orthopedic departmental scientific committee and a 
written informed consent were obtained from all participants before enrollment in the study.

Twenty (20) patients with Adolescent Idiopathic Scoliosis were treated through back methodology by conventional pedicle screw. The patients were agreeable and dependable. The example involved 13 female and 7 male patients with a mean age of $15.3 \pm$ 2.9 years (range 10 - 20 years).

All patients were investigated clinically and radiologically likewise all patients were assessed by SRS 24 survey.

- The inclusion criteria are: Patients with adolescent idiopathic scoliosis. Age: 10-20 years old. Sex: Males and females. Etiology: Idiopathic. No previous surgical intervention for the treatment of scoliosis.

- The exclusion criteria are: Age: more than 20 years old and less than 10 years old. Etiology: Other causes of scoliosis (congenital, traumatic, infantile and juvenile). Patients with associated comorbidity diseases. Previous surgical intervention for the treatment of scoliosis.

- Clinical Evaluation: Initial evaluation focused on history with particular attention to age of the patient, sex, complaints, family history of scoliosis, menarche in girls as an evidence of maturation. Physical examination started with documenting body height, complete general, neurological and trunk examination with documentation of rib hump by measurement of horizontal plumb line (point was placed on the apex of the hump and measurement made between this and a corresponding point on the opposite side of the body in centimeters).

- Radiological Examination: Radiological examination included routine postero-anterior (PA) view and lateral view of full spine in standing position. In addition, right and left bending supine views to assess flexibility of the curves as well as plain X-ray of the pelvis to assess Risser grading.

The PA view for:

Named vertebrae: Apical vertebra, end vertebrae, neutral vertebrae and stable vertebra were determined. Curve pattern: The side of the convexity and site of the curve. Measurement of degree of the curves: by Cobb method. Measurement of apical vertebral rotation (AVR): by Nash and Moe method.

- Evaluation of global balance: Spinal balance: evaluated by measuring the distance between $\mathrm{C} 7$ plumb line and the mid-sacral point. Pelvic balance: evaluated by measuring the angle formed between the line joining between the highest point on both iliac crests and the horizontal line. Shoulder balance: evaluated by measuring T: angle.

- Other measurements: Apical vertebral translation (AVT): distance between the center of apical vertebra and CSVL in lumbar region and 2 line dropped from TI in thoracic curves. Tilt angle of lowest instrumented vertebra (LIVT): the angle between the end plate of LIV and the horizontal line. Adjacent Disc Wedging (ADW): the angle between the lower endplate of LIV and the upper endplate of the distal adjacent vertebra.
Skeletal maturity at the pelvis; the ossification of the iliac crest apophysis evaluated as was originally described by Risser.

\section{The lateral view}

Was used for evaluating the deformity in the sagittal plane. Cobb angles were measured to assess the normal thoracic kyphosis (T5 - T12) and lumbar lordosis (L1 S1). In order to determine structural criteria of the curves as proposed by Link $\boldsymbol{e t}$ al. ${ }^{\left({ }^{(6)}\right.}$ measurement of proximal curve (T2 - T5) and thoracolumbar curve (T10 - L2) was done.

Bending views: Were done to assess flexibility of each curve in order to classify curves and to select fusion levels.

Curve classification: was achieved according to Link et al ${ }^{(6)}$ classification.

Functional evaluation: were done by SRS 24 questionnaire $^{(6)}$.

Operative technique: In all cases, dual rod posterior all pedicle screw constructs were used with rod rotation technique.

Preoperative preparation: All patients were medically assessed as regard medical fitness and laboratory investigation including blood picture, coagulation profile, blood grouping, and at least 3 units of blood were asked.

Preoperative planning: Adequate preoperative planning to save time and effort by detecting strategic vertebrae: upper instrumented vertebra, lower instrumented vertebra, site and number of screws. This was drawn on a paper sheet and placed in a suitable position for all the surgical team to follow thus facilitating smooth execution of the plan.

Patient preparation: The patient received general anesthesia then a urinary catheter was placed to monitor the amount of urine output and the patient was placed prone on a well-padded frame. Care was taken to be sure that the pads were not pressing on brachial plexus, abdomen, and genital organs. The upper extremities were placed in a 90/90 position and pads were placed under the arms as necessary to avoid traction on the brachial plexus. The head was turned to side to avoid possible pressure on the orbit. The patient temperature was preserved through monitoring of the room temperature and application of cotton wraps on the extremities. Prophylactic antibiotics were administrated at induction of anesthesia and if operative procedure prolonged another dose was given. Blood loss was minimized through hypotensive anesthesia which should be started as early as possible (to maintain the mean arterial pressure at $60 \mathrm{mmHg}$ and ranged $50-75$ $\mathrm{mmHg}$ ).

Incision: The operative site was marked for preliminary assessment of the site and extent of skin incision as well as the bony landmarks of rib hump and iliac crests. The skin was then prepared and the patient draped in the usual manner. The skin incision was along a line between $\mathrm{C} 7$ vertebra and the gluteal cleft. A standard posterior midline incision was made from the upper end of the spinous process 2 levels above UIV to the lower end of the lamina 
of LIV. The proximal incision should be long enough to allow convergence of the pedicle screws in UIV. The skin incision didn't follow the line of the spinous processes of the vertebrae in the curve because this will leave an unsightly curved scar that will only accentuate any deformity still present after correction.

Exposure: The spine was exposed by using electrocautery sub-periosteal dissection to reduce bleeding, followed by complete ligament and facet capsule removal. Exposure was extended to the tip of the transverse process to facilitate pedicular screw placement and prepare a suitable bed for bone graft.

Screws placement: Pedicle screws were placed in desired level and going up proximally unless it was difficult to inset a screw so we could skip it. Pedicle screws were inserted on the concave side one by one from the lumbar up to the thoracic segments. Then the screws were inserted to the convex side ranging in every other segment from lower to the uppermost segment after inserting 6 screws in the lower sand uppermost adjacent 3 segment. The first step was to have 8 clear view of the posterior bony elements. In the lumbar spine, the entry site to the pedicle was located at the junction of the superior facet process, pars interarticularis and transverse process. In the thoracic spine, the entry point was $2 \mathrm{~mm}$ inferior and medial to the junction between tie lateral aspect of the superior facet and the superior border of the transverse process. A small lid of cortical bone was removed to reveal underlying cancellous bone. The entry point was penetrated at first by sharp awl then a pedicle finder was gradually advanced into the cancellous bone of the pedicle to avoid penetration of the pedicle cortical wall. The ball tipped probe was used to assure that the canal has a floor and 4 intact walls. The pedicle path was then tapped with an appropriate size which was estimated at the time of probing and was one size smaller than the anticipated screw. It was important to remember that the orientation and configuration of the vertebrae were altered in scoliosis and this should be considered during the placement of the screws. Although it should be avoided if possible, penetration of a side wall was not a problem as long as it was done gently. The ball tipped probe was used to determine which wall was penetrated. Once this determined, the pedicle finder was redirected. In difficult cases K-wires were used. The presumed pedicle entry points are decorticated to facilitate the insertion of the guide pins. Then guide pins are inserted at a depth of $1 \mathrm{~cm}$ through the exposed cancellous bone at the presumed pedicle entry point. Different sizes of $\mathrm{K}$ wires were used on both sides. To facilitate radiograph interpretation the guide pins were directed along the axis of the pedicle in the fontal and sagittal planes. After the guide pins were in place at the planned pedicle screw site, intraoperative PA and lateral roentgenograms were taken to determine the association between the presumed entry point and the ideal entry point identified on the radiograph.

Release: Partial removal of the facet was performed before placement of the rod. The facets included in the fusion were destroyed by inferior facetectomy and removal of the articular cartilage to promote intraarticular fusion. Care should be taken not to disturb the adjacent facets that were not in the fusion area to prevent instability and precocious degenerative change facetectomies encourage a solid fusion and loosen up the spine to aid in correction. We used Ponte osteotomies, which release the posterior column to aid correction in all our cases.

Normalization of the blood pressure: The anesthesiologist was asked to restore the blood pressure to its normal values.

\section{Correction}

Correction side rod placement: The length of the rod was measured and then carefully contoured to restore the normal sagittal dorsal kyphosis and lumbar lordosis. The rod was then inserted following curve, on the concave side, and connected to screws by putting the nuts and left them attached loosely.

Rod rotation: While an assistant firmly press on the convex side downward, the rod was firmly held by the rod holder sand gently rotated 90 degrees to transform the scoliosis into a kyphosis at the thoracic spine and into a lordosis at lumbar spine and restore the sagittal profile in the corrected position. Then preliminary stabilization of the rod was done.

Support side rod placement: The convex side rod was similarly measured, contoured and inserted. More correction was then obtained by in situ bending of the rod or by distraction at the concavity of the curve and compression at the convexity. At the end of this stage, the pedicle screws at either side should be at the same level. It was important to tighten all the nuts at this stage before any further steps were performed using the torque wrench and anti-rotation rod holder.

Debridement: Debridement was performed to remove all the shredded, necrotic tissues. The wound was irrigated with copious amounts of saline solution.

Grafting: Fusion was done by good decortication and bone graft. Part of the fusion was done early during surgery and the major part done later after instrumentation to avoid excessive bleeding. The facets were excised during pedicle screws insertion and the spinous process, laminae and the transverse processes were decorticated after correction by elevating the bone flaps from the laminae and turning the free end of the flaps upward or downward to bring them into contact with the adjacent decorticated laminae. Local bone fragments removed and ribs were cut as small matchstick pieces. If the amount was not sufficient it was supplemented with an autogenous posterior iliac crest graft or bone substitute. In the lumbar region, the graft is placed in the previously prepared posterolateral gutter. In the thoracic region, the graft is placed in the mid-line.

Closure: Closed suction was placed. The deep fascia was tightly closed. The subcutaneous tissue was closed so that tension on the skin was minimized. Skin edges were approximated with suitable sutures.

Operative note: Operative notes were taken regarding the operative time and also the amount of blood loss. Blood loss was calculated by weighting the gauze used intra- 
operatively. This was added to the amount of the blood collected in the suction device.

Postoperative management: Postoperative good analgesia (non-steroidal anti-inflammatory drugs) was used. Laboratory investigations were performed and if the hemoglobin concentration was below $9 \mathrm{gm} \%$ or the hematocrit value was less than $30 \%$, blood transfusion was given. Parenteral antibiotics were given for one week followed by oral for another one week. Good nutrition, chest exercises, and early ambulation were encouraged. The patient was allowed to stand up from second day postoperatively. Dorsolumbar brace was used. The suction drainage was usually removed after 48 hours. Carful observation of the wound was done. Chest tube was removed when pleural drainage was less than $50 \mathrm{ml} / \mathrm{hour}$ and X-ray showed no lung collapse with free costophrenic angles. Postoperative PA and lateral views of dorsolumbar spine were taken while patient standing. The patient's height is measured. Hospital stay was from 5 to 8 days then after which the patient was discharged. The patient returned back after 2 weeks to inspect the wound. All patients were in dorsolumbar brace postoperatively. Brace was worn for 3 months. The patient activities were gradually restored over time. Light activities were allowed from 6 weeks to 3 months postoperatively, and then more activities were allowed without restrictions.

\section{Statistical Analysis}

Data will be collected, revised, coded and entered to the Statistical Package for Social Science (IBM SPSS) version 21 and the following will be done: Qualitative data will be presented as number and percentages while quantitative data will be presented as mean, standard deviations and ranges. The confidence interval was set to $95 \%$ and the margin of error accepted was set to $5 \%$. So, the p-value was considered significant as the following: $\mathrm{P}$ $>0.05$ : Non significant $\quad . \mathrm{P}<0.05$ : Significant.P $<$ 0.01: Highly significant.

\section{RESULTS}

Table (1): Description of LIV \& UIV of studied patients

\begin{tabular}{|c|c|c|c|}
\hline \multicolumn{2}{|c|}{ LIV } & \multicolumn{2}{|c|}{ UIV } \\
\hline Level & Number & Level & Number \\
\hline L1 & 8 & D4 & 5 \\
\hline $\mathrm{L} 2$ & 5 & D5 & 5 \\
\hline L3 & 5 & D3 & 4 \\
\hline $\mathrm{L} 4$ & 2 & D6 & 2 \\
\hline & & D10 & 2 \\
\hline & & $\mathrm{D} 2$ & 1 \\
\hline & & D11 & 1 \\
\hline
\end{tabular}

LIV: L1 vertebra in 8 patients (40\%), L2 in 5 patients (25\%), L3 in 3 patients (15\%) D12 in 2 patients $(10 \%)$ and L4 in 2 patients (10\%). UIV: D4 vertebra in 5 patients (25\%), D5 in 5 patients (25\%), D3 in 4 patients (20\%), D6 in 2 patients (10\%), D10 in 2 patients $(10 \%)$, and D1 in 1 patient $(5 \%)$ and D11 in 1 patient (5\%) (Table 1).
Table (2): Number of fused segments \& screws

\begin{tabular}{|c|c|c|}
\hline & Fused segment & screws \\
\hline Range & $7-13$ & $10-22$ \\
\hline Average & 9.7 & 16 \\
\hline
\end{tabular}

Number of fused segments: ranged from 5 to 13 levels with an average of $9.7 \pm 1.8$ levels per patient. Number of screws: total number of screws was 327 screws ranged between $10-22$ screws with an average of $16 \pm 2.7$ screws (Table 2).

Table (3): Height gain

\begin{tabular}{|c|c|c|c|}
\hline & Preoperative & Postoperative & Gain \\
\hline Range & $133-170$ & $139-176$ & $3-8$ \\
\hline Average & 155.9 & 160.4 & 4.6 \\
\hline
\end{tabular}

The preoperative height of the patients ranged from $133 \mathrm{~cm}$ to $170 \mathrm{~cm}$, with an average of $155.9 \pm 9.3$ $\mathrm{cm}$ while postoperative height ranged between $139 \mathrm{~cm}$ to $176 \mathrm{~cm}$ with an average of $160.4 \pm 9.4 \mathrm{~cm}$. All patients gained height ranged from $3 \mathrm{~cm}$ to $8 \mathrm{~cm}$ with an average of $4.6 \pm 1.1 \mathrm{~cm}(2.9 \%)$ (Table 3 ).

Table (4): Rib hump

\begin{tabular}{|c|c|c|c|}
\hline \multirow{2}{*}{} & \multicolumn{3}{|c|}{ Rib hump } \\
\cline { 2 - 4 } & $\begin{array}{c}\text { Pre- } \\
\text { operative }\end{array}$ & $\begin{array}{c}\text { Post- } \\
\text { operative }\end{array}$ & $\begin{array}{c}\text { \% of } \\
\text { improvement }\end{array}$ \\
\hline Average & 5.8 & 1.3 & $77 \%$ \\
\hline
\end{tabular}

The preoperative rib hump ranged from $3.1 \mathrm{~cm}$ to $9 \mathrm{~cm}$ with an average of $5.8 \pm 1.5 \mathrm{~cm}$ while postoperative rib hump ranged from $0.5 \mathrm{~cm}$ to $2.5 \mathrm{~cm}$ with an average of $1.3 \pm 0.5 \mathrm{~cm}$. Correction ranged from $2.2 \mathrm{~cm}$ to $7.4 \mathrm{~cm}$ with an average of $4.5 \pm 1.3 \mathrm{~cm}$ (about 77\%) (Table 4).

Table (5): Coronal curve magnitude

\begin{tabular}{|r|c|c|c|c|c|c|c|}
\hline & \multicolumn{2}{|c|}{ Preoperative } & Postoperative & \multicolumn{3}{c|}{ Improvement } \\
\hline Curve & Range & Averag & Range & Averag & Rang & Average & $\%$ \\
\hline Thoraci & $45-83$ & 55.6 & $0-25$ & 8.1 & $37-58$ & 46 & 82.7 \\
\hline Lumbar & $55-68$ & 62.7 & $5-13$ & 9.7 & $50-55$ & 53 & 84.6 \\
\hline Th/L & $40-75$ & 47.1 & $0-23$ & 6.3 & $37-52$ & 40.9 & 86.6 \\
\hline Primary & $40-83$ & 53.7 & $0-25$ & 7.6 & $37-58$ & 46.1 & 83 \\
\hline
\end{tabular}

Curves ranged preoperatively between $40^{\circ}$ to $83^{\circ}$, with an average of $53.7^{\circ} \pm 9.3$. While postoperatively coronal curve magnitude ranged from $0^{\circ}$ to $25^{\circ}$ with an average of $7.6^{\circ} \pm 6.5$. Improvement ranged between $37^{\circ}$ $58^{\circ}$ with an average of $46.1^{\circ} \pm 4.8$. Percentage of improvement was about $83 \%$.

Coronal Cobb angle of thoracic curves ranged from $45^{\circ}$ to $83^{\circ}$ with an average of $55.6^{\circ} \pm 8$. While postoperatively it ranged from $0^{\circ}$ to $25^{\circ}$ with an average of $8.10 \pm 5.8$. Improvement ranged between $37^{\circ}-58^{\circ}$ with an average of $46^{\circ} \pm 4.9$ of about $82.7 \%$.

Coronal Cobb angle of lumbar curves ranged from $55^{\circ}$ to $68^{\circ}$ with an average of $62.7^{\circ} \pm 6.8$. While postoperatively it was ranged from $5^{\circ}$ to 139 with an average of $9.7^{\circ} \pm 4$. Improvement ranged between $50^{\circ}$ $55^{\circ}$ with an average of $53 \pm 2.7$ of about $84.6 \%$. 
Also coronal Cobb angle of thoracolumbar curves ranged from $40^{\circ}$ to $75^{\circ}$ with an average of $47.1^{\circ} \pm 12.9$. While postoperatively it ranged from0 to $23^{\circ}$ with an average of $6.3^{\circ} \pm 9$. Improvement ranged between $37^{\circ}$ $52^{\circ}$ with an average of $40.9 \pm 5$ of about $86.6 \%$ (Table 5).

Table (6): Coronal curve groups.

\begin{tabular}{|c|c|c|c|c|c|c|c|}
\hline & \multicolumn{2}{|c|}{ Preoperative } & \multicolumn{2}{c|}{ Postoperative } & \multicolumn{3}{c|}{ Improvement } \\
\hline Group & Range & Average & Range & Average & Range & Average & $\%$ \\
\hline $\mathbf{1}$ & $40-58$ & 49.1 & $0-13$ & 5.2 & $37-50$ & 43.4 & 87 \\
\hline $\mathbf{2}$ & $60-83$ & 66.4 & $10-25$ & $15-9$ & $42-58$ & 50.5 & 75 \\
\hline
\end{tabular}

Group 1 ranged preoperatively from $40^{\circ}$ to $58^{\circ}$ with an average of $49.1^{\circ} \pm 5.2$ while postoperatively it ranged from $0^{\circ}-13^{\circ}$ with an average of $5.2^{\circ} \pm 4.8$. It improved with an average of $43.4^{\circ}$ ranged between $37^{\circ}$ $50^{\circ} \pm 3.4$. Percentage of improvement was about $87 \%$.

While group 2 ranged from $60^{\circ}$ to $83^{\circ}$ with an average of $66.4^{\circ} \pm 6.9$ while postoperatively it ranged from $10^{\circ}-25^{\circ}$ with an average of $15.9^{\circ} \pm 4.8$. It improved with an average of $50.5^{\circ} \pm 3.7$ ranged between $42^{\circ}-58^{\circ}$. Percentage of improvement was about $75 \%$ (Table 6).

Table (7): Primary coronal curve

\begin{tabular}{|c|c|c|c|c|c|c|c|}
\hline & Preop. & Postop. & $\begin{array}{c}\text { Improve } \\
\text { ment }\end{array}$ & & F.U & Loss & \\
\hline & Average & Average & Average & $\%$ & Average & Average & $\%$ \\
\hline Curve & 53.7 & 7.6 & 46.1 & 83 & 9.5 & 1.9 & 3.6 \\
\hline
\end{tabular}

After 9 month, curves ranged from $0^{\circ}-30^{\circ}$ with an average of $9.5^{\circ} \pm 7$. While loss of correction ranged from $0^{\circ}-8^{\circ}$ with an average of $1.9 \pm 2.2$. Percentage of loss of correction after 9 month was about $3.6 \%$ (Table 7).

Table (8): Thoracic kyphosis

\begin{tabular}{|c|c|c|c|c|c|c|c|}
\hline \multirow{2}{*}{} & \multicolumn{2}{|c|}{ Preoperative } & \multicolumn{2}{|c|}{ Postoperative } & \multicolumn{3}{|c|}{ Improvement } \\
\cline { 2 - 8 } & Range & Average & Range & Average & Range & Average & $\%$ \\
\hline Hypo & $5-9$ & 6.6 & $25-32$ & 29.3 & $16-27$ & 12.5 & 75 \\
\hline Neutral & $25-36$ & 32.5 & $26-35$ & 30.3 & $-6-5$ & -2.2 & 6.7 \\
\hline Hyper & $42-70$ & 49 & $25-37$ & 30 & $9-38$ & 18.9 & 38 \\
\hline
\end{tabular}

Thoracic kyphosis ranged preoperatively between $5^{\circ}-70^{\circ}$ with an average of $30.5^{\circ}+14.2$. Postoperative thoracic kyphosis ranged between $25^{\circ}-37^{\circ}$ with an average of $29.8^{\circ}+2.7$. The change ranged from $38^{\circ}$ to $15^{\circ}$ with an average of $0.1^{\circ}+14$ about $0.3 \%$.

The sagittal modifier was hypokyphotic (-) in 9 (45\%) patients, ranged between $5^{\circ}-9^{\circ}$ preoperatively with an average of $6.6^{\circ} \pm 1.6$ while postoperatively ranged between $25^{\circ}-32^{\circ}$ with an average of $29.3^{\circ} \pm 2.2$. Improvement ranged between $16^{\circ}-27^{\circ}$ with an average of $12.5^{\circ} \pm 2.3$. Percentage of correction was about $75 \%$.

Thoracic kyphosis was neutral $(\mathrm{N})$ in $6(30 \%)$ patients ranged from $25^{\circ}$ to $36^{\circ}$ with an average of $32.5 \pm$ 3 while postoperatively ranged from $26^{\circ}$ to $35^{\circ}$ with an average of $30.3^{\circ} \pm 2.3$ within normal kyphosis. Changes ranged from $6^{\circ}$ to $5^{\circ}$ with an average of $2.2+3(6.7 \%)$.

There were $5(25 \%)$ patients with hyperkyphosis preoperatively ranged between $42^{\circ}-70^{\circ}$ with an average of $49^{\circ} \pm 8$ while postoperatively ranged between $25^{\circ}-37^{\circ}$ with an average of $30^{\circ} \pm 13.5$. The angle was decreased with an average of $18.9^{\circ}$ ranged between $9^{\circ}-38^{\circ} \pm 8.3$ with $38 \%$ correction (Table 8 ).

Table (9): Sagittal curves (range \& average in degrees).

\begin{tabular}{|c|c|c|c|}
\hline & Preoperative & Postoperative & Follow up \\
\hline Th. & $5-70$ & $25-37$ & $37-37$ \\
Kyphosis & $(30.5)$ & $(29.8)$ & $(31.3)$ \\
\hline L. Lordosis & $5-58(44.7)$ & $5-50(43.7)$ & $9-50(45.2)$ \\
\hline
\end{tabular}

Curves ranged preoperatively between $35^{\circ}-58^{\circ}$ with an average of $44.7^{\circ} \pm 6.6$ while postoperatively ranged between $35^{\circ}-50^{\circ}$ with an average of $43.7^{\circ} \pm 3$. The change ranged from $15^{\circ}$ to $10^{\circ}$ with an average of $1^{\circ} \pm$ 5.8 about $2.3 \%$,

After 9 month curves ranged between $39^{\circ}-50^{\circ}$ with an average of $45.2^{\circ} \pm 2.2$. The change ranged from $6^{\circ}$ to $8^{\circ}$ with average of $1.4^{\circ} \pm 3.3$ about $3.2 \%$ (Table 9).

Table (10): Lumbar lordosis

\begin{tabular}{|c|c|c|c|}
\hline & Preoperative & Postoperative & Follow up \\
\hline Hypo & 36.6 & 41.8 & 44.8 \\
\hline Normal & 47 & 44.3 & 45.2 \\
\hline
\end{tabular}

There were $11(20 \%)$ patients preoperatively with lumbar lordosis ranged from $35^{\circ}$ to $39^{\circ}$ (hypolordotic) with an average of $36.6^{\circ} \pm 1.6$ while postoperatively ranged between $40^{\circ}-45^{\circ}$ with an average of $41.8^{\circ} \pm 2$. Improvement ranged between $1^{\circ}-10^{\circ}$ with an average of $5^{\circ} \pm 2.5$, about $14 \%$. After 9 month lordosis was between $40^{\circ}-49^{\circ}$ with an average of $44.8^{\circ}+2.7$ with change ranged from $2^{\circ}$ to $8^{\circ}$ with an average of $3^{\circ} \pm 3.6$ about $7 \%$.

There were $16(80 \%)$ patients with normal lordosis ranged from $40^{\circ}$ to $58^{\circ}$ with an average $47^{\circ} \pm 5.6$ while postoperatively ranged between $35^{\circ}-50^{\circ}$ with an average of $44.3^{\circ} \pm 3$. The change ranged from $15^{\circ}$ to $6^{\circ}$ with an average of $2.8^{\circ} \pm 5.2$, about $6 \%$,

After 9 month lordosis was between $39^{\circ}-50^{\circ}$ with an average of $45.2^{\circ} \pm 2$. Lordosis increased by an average of $1^{\circ} \pm 3$ about $0.2 \%$ ranged from $6^{\circ}$ to $7^{\circ}$ (Table 10 ).

Table (11): Primary curve rotation.

\begin{tabular}{|c|c|c|c|c|}
\hline & \multicolumn{2}{|c|}{ Preoperative } & \multicolumn{2}{c|}{ Postoperative } \\
\hline Grade & Number & $\%$ & Number & $\%$ \\
\hline I & 3 & $15 \%$ & 14 & $70 \%$ \\
\hline II & 9 & $45 \%$ & 4 & $20 \%$ \\
\hline III & 8 & $40 \%$ & 0 & 0 \\
\hline 0 & 0 & 0 & 2 & $10 \%$ \\
\hline
\end{tabular}

Rotation of primary coronal curves ranged preoperatively between $1-3$ grades according to Nash \& Moe with an average of $22 \pm 0.7$ while postoperatively ranged between $0-2$ grades with an average of $1.12 \pm 0.6$. Improvement was ranged between $0-2$ grades with an average of $1 \pm 0.6$. Correction was about $48 \%$.

After 9 month there was no significant loss of amount of correction obtained. Preoperative, there were 3 patents with grade 1,9 patients were grade 2 , and 8 
patients were grade 3. Postoperatively there were 14 patients with grade 1, 4 patients were grade 2, 2 patients were grade 0 and none grade 3 (Table 11).

\section{DISCUSSION}

AIS is a structural 3-dimensional deformity of the spine that occurs in otherwise healthy children during puberty. It is the most common form of scoliosis and the diagnosis is made by exclusion. Significant controversy exists regarding the natural history of untreated AIS, the value of surgical correction and the ideal surgical instrumentation ${ }^{(7)}$.

The goals of surgical treatment for AIS are to arrest progression by achieving a solid fusion, correction of the deformity, improvement of the cosmetic appearance, improvement of the functional outcomes, physical and psychosocial health and diminishing the development of low back pain, degenerative changes, functional impairment and cardiopulmonary compromise in adulthood. Surgical correction of AIS is considered for curves greater than $45^{\circ}$ in immature patients and for curves greater than $50^{\circ}$ in mature patients. Surgery is usually performed during adolescence, but newer techniques allow good creation to be accomplished into early adulthood ${ }^{(8)}$.

The treatment of AIS has undergone a significant evolution in the past decades. Traditionally, Harrington instrumentation. Luque rods, and sublaminar wires were the mainstay of treatment. However, since the advent of 3rd generation Cotrel-Dubousset instrumentation, the use of segmental fixation has become the main option ${ }^{(9)}$. The spine is corrected with a combination of rods, hooks, screws and wires while being fused by bone graft either from the patient or artificially. Hooks have been widely used in the treatment of AIS. Hook insertion techniques are standard, familiar, and generally accepted. However, hook displacement or pullout may occur during curve reduction maneuvers. Hooks are effective at distraction and compression, but lateral translation and derotation are difficult to achieve. By definition all hooks intrude into the spinal canal and may be associated with neurologic complications (10). Sublaminar wiring with posterior instrumentation is one of the methods used when long fusions involving 10-12 thoracolumbar levels are required. Classically, wires are used at every consecutive level to make the construct as rigid as possible, although complications like dural tears, cerebrospinal fluid leak, and neurological deficit have been reported during their passage. ${ }^{(11)}$

These previous instrumentation methods were fraught with complications including loss of fixation, dislodgement of implants, flat-back syndrome and suboptimal fixation. Another concern with older techniques was the inability to obtain curve correction in all 3 planes. Pedicle screws have been used for the management of deformities since 1988. Initially, pedicle screws were used in the thoracolumbar spine where the pedicles were large and easily visualized. With the success in the thoracolumbar spine, pedicle screws were gradually used in the more proximal levels
${ }^{(9)}$. The use of pedicle screw implants for the treatment of AIS has gained popularity over the past several years. Pedicle screws have shown superior biomechanical properties over other instrumentation techniques of the spine. The use of pedicle screws has also demonstrated the ability to fuse fewer motion segments of the spine, thus preserving motion caudal to the fusion. This also should theoretically decreases the stress placed on distal segments preventing subsequent disc degeneration ${ }^{(12)}$.

Pedicle screw fixation appears to provide the surgeon with improved ability to correct the 3dimensional deformity present in AIS. Advocates of pedicle screw constructs report that the advantages for all pedicle screw constructs in AIS include improved coronal, sagittal, and rotational correction, lower pseudartrosis rates, lower implant failures and fewer postoperative bracing requirements when compared with conventional hook and wire constructs. Pedicle screws allow for three-column fixation of the vertebral body, thereby allowing for improved correction over hook constructs and decreased rate of loss of correction. Additionally they allow for true de-rotation of the spine. All pedicle screws constructs have an important advantage in that vertebrae can be de-rotated directly, thus reducing vertebral rotation and reducing thoracic humps ${ }^{(13)}$.

Pedicle screw constructs also allow the surgeon to perform posterior-only surgeries for deformities traditionally treated with a circumferential approach. Recently, Luhmann et al. ${ }^{(14)}$ showed that in curves between $70^{\circ}-100 \%$ the use of all pedicle screw contracts allowed the surgeons to perform a posterioronly surgery with minimal coronal correction difference when compared with a combined anterior and posterior procedure. With the morbidity associated with anterior thoracic and lumbar surgeries, a posterior only approach is a viable and perhaps preferable option, which still affords great correction power with minimal associated morbidity. All pedicle screw constructs also facilitate osteotomies, including Ponte and pediclesubtraction osteotomies and posterior vertebral column resection, 50 that even rigid and severe curves can be corrected efficiently without anterior procedures ${ }^{(14)}$.

The use of pedicle screws also has advantages over hook and wire implants with respect to vertebral anatomy. When placed optimally, screws are completely external to the spinal canal. The low profile head design of most screw implants facilities segmental fixation, which is more difficult to accomplish with hooks. Also, facet joints, lamina, and transverse processes are free of implants such as wires or hook blades. Leaving more surface area available for aggressive de-cortication and grafting without destabilizing the instrumentation. All pedicle screw constructs also obviate the need for an autologous iliac bone graft. In most patients with AIS, this is replaced by a graft of local bone, with or without bone extenders (13).

There is experimental as well as clinical evidence to suggest that all pedicle screw constructs may be stiff 
enough to prevent the crankshaft phenomenon. Traditionally, this complication has been prevented by performing surgery with dual anterior and posterior approaches in at-risk patients. However, a posterioronly approach greatly diminishes morbidity in these patients ${ }^{(15)}$. Thus, all pedicle screw constructs are presently the most popular posterior surgical methods for treating AIS, while, as in conventional scoliosis surgery, the meticulous posterior release of the facet joints, ligaments remains important. Despite these advantages, all pedicle screw constructs have several drawbacks. These include a steep learning curve, difficulties associated with accurately placing pedicle screws within deformed spine, the potential for neurovascular injuries due to screw malposition and higher instrumentation costs ${ }^{(16)}$.

Clinical and radiological results of 20 patients with AIS corrected with all pedicle screw constructs were presented.

\section{Primary curve correction:}

All pedicle screw constructs provide comparable or better curve correction in comparison to other constructs. Westrick et al. ${ }^{(17)}$ reported in 5 to 20 years evidencebased study on surgical results of treatment of AIS that anterior systems gave an overall average correction of $62.4 \%$, Harrington rods gave a $34.1 \%$ average correction and segmental hooks gave a $51.2 \%$ average correction. The study using Wisconsin fixation reported $39 \%$ correction, whereas the highest percent correction obtained in the pedicle screw and Isola hybrid patients, about $69.5 \%$ and $63 \%$ respectively ${ }^{(17)}$. A direct comparison between the hybrid and all pedicle screw constructs revealed superior primary curve correction in all-screw group (76\% versus $50 \%)$ in 58 AIS patients as reported by Kim et al. ${ }^{(18)}$. This is consistent with a previous study using the Moss-Miami hybrid construct in 61 patients, which revealed a $56 \%$ correction rate of the primary curve.

In our study, a primary coronal curve correction of $83 \%$ was achieved with a preoperative flexibility index of $48 \%$. This correction rate is comparable with those of other studies on segmental pedicle screw fixation of AIS as reported earlier by Suk et al. ${ }^{(19)}$ in which they evaluated 203 patients with AIS. They obtained 72\% correction of the primary coronal curve compared to $55 \%$ with hooks.

Also in our study we achieved better correction of the coronal curves less than $60^{\circ}$ of about $87 \%$ while we obtained $75 \%$ correction in curves greater than $60^{\circ}$. We noticed that no effect of age on average curve correction. Comparison of curve correction between males and females revealed no significant difference. We noticed that better correction was obtained in thoracolumbar curves of about $86.6 \%$ followed by lumbar curves of about $84.6 \%$ then thoracic curves of about $82.7 \%$. There was a strong positive correlation between the preoperative flexibility index of the primary curves and percentage of postoperative correction of these curves.
Correction loss of the major curve with various types of instrumentation has been given great attention in the literature. In the same study by Westrick $\boldsymbol{e t}$ al. (17) loss of correction averaged $11 \%$ for anterior constructs, $17.5 \%$ for Harington rods, $6.5 \%$ for segmental hooks, $3.4 \%$ for pedicle screw fixation, $3 \%$ for Isola hybrid and $7 \%$ for Wisconsin fixation.

Modern instruments used in scoliosis surgery have decreased the correction loss rate in a study by Liljenqvist et $\boldsymbol{a l}$. (20) for example, the use of cotreldubusset instrumentation in 64 patients produced an average correction loss of $5.2^{\circ}(11.6 \%)$. Wu et al. (24) exported correction loss of about $8.3 \%$ in all screw group compared to $14 \%$ loss of correction in hook group. The use of pedicle screws, which can provide better holding power, can substantially improve the correction loss rate from 3-6\%. In our study at the end of follow- up, the correction loss averaged $1.9^{\circ}(3.6 \%)$.

\section{Sagittal plane correction:}

The effect of pedicle screw constructs on sagittal plane correction is not clear. Patients with AIS often display hypokyphosis in the thoracic region which surgery would address. Suk et al. ${ }^{(21)}$ found better improvement of hypokyphosis with pedicle screws than with hooks. They used rod rotation technique and a very stiff 7-mm stainless steel rod. However, Kim et al. ${ }^{(18)}$ reported a decrease in thoracic kyphosis in patients treated with pedicle screw constructs. Recently, Clement et al. ${ }^{(22)}$ reported improved kyphosis in hypokyphotic patients treated with pedicle screw constructs. Improved sagittal correction may be surgeon-, positioning-, or technique-dependent. Further research is needed on the effect of instrumentation and fixation type on hypokyphosis.

In our study, the average correction of thoracic hypokyphosis in 9 patients was $75 \%$ (ranged between $25^{\circ}$ - $32^{\circ}$ ). Also thoracic hyperkyphosis in 5 patients was corrected by about $38 \%$ ranged between $25^{\circ}-37^{\circ}$. We obtained thoracic kyphosis ranged between $27^{\circ}-37^{\circ}$ without loss of correction at the end of follow-up. The results of Harrington rod instrumentation for correction of AIS highlight the importance of preserving or restoring sagittal alignment of the spine. Numerous reports have pointed out the complications of significant loss of lumbar lordosis, which results in flat-back syndrome ${ }^{(23)}$.

In our study, lumbar lordosis ranged within normal measures between $35^{\circ}-50^{\circ}$ postoperatively and well maintained at the last follow.

\section{Apical vertebral rotation:}

Another inherent benefit of pedicle screw fixation is the ability to correct the spine in a three-dimension. The use of a simple rod de-rotation maneuver as a standalone method of de-rotation or coupled with a direct vertebral rotation bas recently gained in popularity because of the ability of pedicle screws obtaining purchase of all 3 columns of the vertebral body ${ }^{(13)}$. In our study, we did not perform direct vertebral rotation. Instead, we used rod rotation technique. With this technique a $48 \%$ correction of primary curve rotation 
was achieved without loss of correction at the end of follow-up. This is comparable with the results reported by Wu et $\boldsymbol{a l} .{ }^{(24)}$. They evaluated 168 patients using the same technique and reported correction of about $46 \%$ of apical vertebral rotation and compared this to hook constructs in which correction was $39 \%$.

In our study, the spontaneous improvement of clinical rib hump of $77 \%$ was achieved with this technique.

\section{Global balance:}

In our study, we obtained improved global balance as regards spinal, shoulder and pelvic balance by about $87 \%, 73 \%$ and $83 \%$ respectively which is comparable with other studies as reported by Stephen-Richard $\boldsymbol{e t}$ al. ${ }^{(25)}$

Apical vertebral translation (AVT), Tilt angle of lowest instrumented vertebra (LIVT) and Adjacent disc wedging (ADW):

There were some reports conceding the changes in AVT, LIVT, or ADW. Liljenqvist et al. ${ }^{(20)}$ found that the correction of both AVT and LIVT was significantly greater in the all pedicle screw group compared to the hook group (AVT $64 \%$ vs. 54\% and LIVT $70 \%$ vs. $60 \%$ ). Shuflebarger et al. ${ }^{(26)}$ found $81 \%$ of LVT correction with all pedicle screws. They did not have a hook comparison group in their study. They stated that better horizontalization of LIV can be achieved with screw constructs because the more lateral position of the pedicle screw provides a considerably better leverage and because the tangential fixation strength of pedicle screws is significantly greater than that of the laminar hooks.

A good correction of AVT, LIVT, and ADW (about $85.5 \%, 81.5 \%$, and $79.5 \%$ improvement respectively) with minimal loss of correction at the end of follow-up was achieved in our patients.

\section{Patient Outcome Scores:}

Luimana et al. ${ }^{(14)}$ noted that AIS patients treated by all pedicle screws constructs had significantly better scores as regard postoperative self-image and function.

In our study assessment of the patients functionally was done using SRS 24 Questionnaire. We found that total score ranged from 96-104 with an average of 100.2 of total score 120 , representing good outcome. Percentage of score ranged between $80 \%-85 \%$ with an average of $83.52 \%$ (of percentage $100 \%$ been the best and $20 \%$ been worst). Mean score ranged between 4 4.25 with an average of 4.17 (of mean score 5 representing best and 1 been worst). This was better than total score reported by Kan et al. ${ }^{(27)}$ of about $93 \pm$ 18 points.

\section{Complications:}

There was one case $(5 \%)$ of dural penetration during owling managed conservatively. Also, there was one case of pleural tear $(5 \%)$ managed conservatively.
We did not have a neurological complication, a metal breakage or hardware prominence need revision.

\section{Implant density:}

Insertion of screws in every single vertebra is not necessary as a satisfactory correction can be achieved with a low implant density of average 1 screw per vertebra $(50 \%){ }^{(28)}$.

In our study, we achieved good correction of the deformity with implant density about $60.8 \%$. Total number of screws in our study was 327 screws ranged between 10-22 screws/case with average about 16 screws. We obtained about $83 \%$ correction, which was higher than obtained by Omar $\boldsymbol{e t}$ al. ${ }^{(29)}$. They reported correction of about $70 \%$ after they used about 19.5 screws/case ranged between 15 - 26 screws in fusing average 12.3 vertebrae $(10-15)$ while in our study number of fused segments was 9.8 ranged between 7 13 levels.

\section{Operative time and blood loss:}

In our study, operative time was 3.3 hours (2.5- 5), which is less than that reported by Omer et al. ${ }^{(29)}$. They treated 22 patients all with pedicle screw constructs. Operative time in their study was 5.5 hours (5- 6.5) compared to hook constructs, which was 6 hours (5-7). The average amount of blood toss in our study was 475 $\pm 236 \mathrm{ml}(650$ - 1150), which was less than that reported in the same study by Omar et al. ${ }^{(29)}$ depending on surgical and anesthetic techniques.

\section{LIMITATIONS}

Limitations of our study included relatively small sample size and no comparative groups. We also did not use CT scan to assess the accuracy of screw placement. Also longer period of follow-up is needed. However, our study demonstrated the early experience and clinical and radiological results of using all pedicle screw constructs in treatment of AIS in our Department of Orthopedic Surgery in Al-Azhar University.

\section{CONCLUSION}

Finally, we concluded that all pedicle screw constructs are reliable in the treatment of AIS, while longer follow up period is needed.

\section{REFERENCES}

1. Terminology Committee of the Scoliosis Research Society (1976): A glossary of terms. Spine, 1: 57-8.

2. Lonstein JE (1994): Adolescent idiopathic scoliosis. Lancet, 344: 8934.

3. Parent S, Newton PO, Wenger DR (2005): Adolescent idiopathic scoliosis: etiology, anatomy, natural history, and bracing. Instructional Course Lectures, 54: 529-36.

4. Mo F, Cunningham ME (2011): Pediatric scoliosis. CurrRev Musculoskelet Med., 4: 175-182.

5. Miller DJ, Vitale MG, Hibbs RA et al. (2015): Pioneer of spinal fusion. Spine, 40: 1311-1313.

6. Link T, Hackenberg L, Liljenqvist U (2002): Axial and tangential fixation strength of pedicle screws versus hooks in the thoracic spine in relation to bone mineral density. Spine, 27: 937-942. 
7. Rinsky LA, Gamble JG (1988): Adolescent idiopathic scoliosis. West J Med., 148: 182-191.

8. Janicki JA, Allman B (2007): Scoliosis: Review of diagnosis and treatment. Pediatric Child Health, 12 (9): 771-776.

9. Matsumoto M, Hosogane N, Toyama Y, Watanabe $K$ (2014): Updates on surgical treatments for pediatric scoliosis. Journal of Orthopaedic Science, 19 (1): 6-14

10. Ashraf SA, Jan S, Khai SL (2006): Pedicle Screws Versus Pedicle Hooks for Correction of Thoracic Scoliosis Using the Universal spinal System II. A Modification of the Original Technique. EJ.NS., 21 (2): 123-133.

11. Gadgil EA, Rahmatalla A, Dove J, Maffulli $\mathbf{N}$ (2002): A study of the mechanical stability of scoliosis constructs using variable numbers of sublaminar wires. Eur Spine J., 11: 321-326.

12. Lehman RA, Lenke LG, Keeler KA, Kim YJ, Buchowski JM, Cheh G, Kuhns CA, Bridwell KH (2008): Operative treatment of adolescent idiopathic scoliosis with posterior pedicle screw-only constructs: minimum three-year follow-up of one hundred fourteen cases. Spine, 33 (14): 1598-604.

13. Kishore M, Angeliki P, Christopher WR (2007): Evidence-Based Medicine Analysis of All Pedicle Screw Constructs in Adolescent Idiopathic Scoliosis. Spine, 32 (19): S109-S114.

14. Luhmann SJ, Lenke LG, Kim YJ et al. (2005): Thoracic adolescent idiopathic scoliosis curves between $70^{\circ}$ and $100^{\circ}$. Is anterior release necessary? Spine, 30: 2061-7.

15. Esteban C (2009): Use of All-pedicle-screws constructs in treatment of adolescent idiopathic scoliosis. J Am Acad Orthop Surg., 17: 550-561.

16. Sud A, Tsirikos AI (2013): Current concepts and controversies on adolescent idiopathic scoliosis: Part 2. Indian J Orthop., 47: 219-29.

17. Westrick W, Edward R, Timothy W (2011): Adolescent Idiopathic scoliosis: S-Year to 20-Year Evidence-based Surgical Results. Pediatric Orthopedic, 31: S61-S68.

18. Kim YJ, Lenke LG, Kim J, Bridwell KH, Cho SK, Cheh G (2006): Comparative analysis of pedicle screw versus hybrid instrumentation in posterior spinal fusion of adolescent idiopathic scoliosis. Spine, 31 (3): 291-298.

19. Suk SI, L.ee CK, Kim WJ, Chung YJ, Park YB (1995): Segmental pedicle screw fixation in the treatment of thoracic idiopathic scoliosis. Spine, 20: 1399-1405.

20. Liljenqvist $\mathrm{U}$, Lepsien $\mathrm{U}$, Hackenberg $\mathrm{L}$ et al. (2002): Comparative analysis of pedicle screw and hook instrumentation in posterior correction and fusion of idiopathic thoracic scoliosis. Eur Spine J., 11: 33643 .

21. Suk SI, Kim WJ, Kim JH, Lee SM (1999): Restoration of thoracic kyphosis in the hypokyphotic spine: a comparison between multiple-hook and segmental pedicle screw fixation in adolescent idiopathic scoliosis. J Spinal Disord., 12: 489495.

22. Clement JL, Chau E, Kimkpe C, Vallade MJ (2008): Restoration of thoracic kyphosis by posterior instrumentation in adolescent idiopathic scoliosis: Comparative radiographic analysis of two methods of reduction. Spine, 33: 1579- 1587.

23. Lagrone MO, Bradford DS, Moe JH, Lonstein JE, Winter RB, Ogilvie JW (1988): Treatment of symptomatic fatback after spinal fusion. J Bone Joint Surg Br., 70 (4): 569-580.

24. Wu X, Yang S, Xu W, Yang C, Ye S, Liu X, Li J, Wang J (2010): Comparative intermediate and longterm results of pedicle screw and hook instrumentation in posterior correction and fusion of idiopathic thoracic scoliosis. Clinical Spine Surgery, 23 (7): 467-73.

25. Richards BS, Herring JA, Johnston CE, Birch JG, Roach JW (1994): Treatment of adolescent idiopathic scoliosis using Texas Scottish Rite Hospital instrumentation. Spine, 19(14):1598-605.

26. Shuflebarger HL, Geck MJ, Clark CE (2004): The posterior approach for lumbar and thoracolumbar adolescent idiopathic scoliosis: posterior shortening and pedicle screws. Spine, 29: 26976.

27. Kan M, Christoph S, Mazda F (2012): Posterior correction of thoracic adolescent idiopathic scoliosis with pedicle screw instrumentation results of 48 patients with minimal 10-year follow up. Eur Spine J., 25: 33-3.

28. Suk S, Lee SM, Chung ER et al. (2005): Selective thoracic fusion with segmental pedicle screw fxation in the treatment of thoracic idiopathic scoliosis: more than S-year follow-up. Spine, 30: 1602-9.

29. Omar K, Unay K, Tezer M, Ozturk C, Aydogan M, Mirzanli C (2008): Comparative analysis of pedicle screw versus hybrid instrumentation in adolescent idiopathic scoliosis surgery. International $\begin{array}{llll}\text { orthopaedics, } & 32 & \text { (4): } & \text { 523-8. }\end{array}$ 